\title{
Chronic Obstructive Pulmonary Disease: Proprioception Exercises as an Addition to the Rehabilitation Process
}

\author{
Bruno Bordoni ${ }^{1}$, Marta Simonelli ${ }^{2}$ \\ 1. Physical Medicine and Rehabilitation, Foundation Don Carlo Gnocchi, Milan, ITA 2. Integrative/Complimentary \\ Medicine, French-Italian School of Osteopathy, Pisa, ITA
}

Corresponding author: Bruno Bordoni, bordonibruno@hotmail.com

\begin{abstract}
Respiratory rehabilitation in patients with chronic obstructive pulmonary disease (COPD) is recognized as a cornerstone for the therapeutic path. Physiotherapy involves physical activity with aerobic and anaerobic exercises, which can improve the patient's symptomatic picture, such as motor function, emotional status (depression and anxiety), and improve the pain perception. The training of proprioception is not included in the structure of the exercises proposed by the Global Initiative for Chronic Obstructive Lung Disease (GOLD). The training of proprioception is a very useful strategy for stimulating the cerebellum, a neurological suffering area in patients with COPD. The cerebellum sorts information about pain and emotions, as well as motor stimuli. The article discusses the need to introduce proprioception in respiratory rehabilitation protocols, highlighting the neurological relationships with the management of comorbidities.
\end{abstract}

Categories: Physical Medicine \& Rehabilitation, Pulmonology, Osteopathic Medicine Keywords: copd, pain, depression, diaphragm, physiotherapy, rehabilitation, fascia

\section{Introduction And Background}

Chronic obstructive pulmonary disease (COPD) is the expression of multiple local and systemic disorders that culminate in chronic respiratory diseases and chronic airflow limitation; chronic cough, dyspnea, phlegm production and recurrent infections or inflammations of the bronchial tree [1,2]. Statistical projections highlight an increase in world mortality that is around 45 million deaths in the next 30 years [2]. COPD is the third cause of mortality in the world and an estimated 400 million people are affected by this chronic disease; about one in five patients, once discharged from the hospital, is still hospitalized within 30 days $[3,4]$. The best-known causes are the consumption of tobacco (active and/or passive), genetic predisposition, air pollution, working environment that subjects people to breathe toxic substances and/or to work in cold and humid environments [1,5]. COPD patients are subject to different comorbidities. A patient with COPD is unlikely to be free from comorbidities; in particular, if the pathology is long-lasting; metabolic, cardiovascular, neurological, psychological and psychiatric systemic diseases occur [6,7]. There is a decrease in the efficiency of neuromotor coordination, a decrease in muscle mass with phenotypic changes, a functional and morphological alteration of the diaphragm muscle and the presence of tongue disorders at night [8-10]. There is a direct relationship between muscle strength and peak inspiratory flow rate and an inverse relation between the severity of symptoms and functional capacity (physical training) $[11,12]$. Despite a suboptimal adherence to the physiotherapy program to improve the overall performance of the patient, we know that there is evidence on the usefulness of sending the person to carry out physical activity by health professionals (physiotherapists) [13]. Physical rehabilitation allows to improve the symptomatic picture (dyspnea and fatigue), the emotional sphere, and the quality of life (QoL) [14]. Respiratory rehabilitation through exercise is a cornerstone for the patient's path, as highlighted by the 2019 Global Initiative for Chronic Obstructive Lung Disease (GOLD) report $[15,16]$. The recommended physical activity is aerobic and anaerobic or mixed, depending on the study examined [17-19]. Another approach includes balance exercises, as a maintained stand on one leg or get up and sit down from a chair; the rationale is based on the fact that many COPD patients have problems of balance and fear of falling [20,21]. The causes of this inability to maintain a correct posture (daily movements) have no direct relationship with the severity of respiratory pathology (FEV1) [22]. A study has shown a causality linked to central neurological factors and the presence of a cognitive loss, probably due to cerebral vascular lesions. Impaired neuromuscular coordination, cognitive impairment may adversely affect postural control. Another element that negatively affects the functionality of the movement and the emotional status is the presence of chronic pain in these patients [23]. Pain is often localized in the chest area and in the back; pain is often not related to the presence of angina but is multifactorial [24-27]. There is a triad that worsens the patient's health status: chronic pain, depression and anxiety, and difficulty in movement [28]. The aim of the article is to highlight the need to use proprioceptive exercises in the usual rehabilitation program of COPD patients, reviewing the scientific literature about the connection between the cerebellum, emotions and perceived pain. The information we have suggests the insertion of specific exercises for the stimulation of proprioception, as it could improve the clinical picture related to pain and depression, with positive effects on muscle movement. The article could be considered to implement the physiotherapeutic approach. To 


\section{Cureus}

conclude, the initiative could be a tool for post-COVID-19 patients in the near future; a hypothesis that still lacks data to make a statement.

\section{Review}

\section{Epidemiology of chronic pain, depression, and anxiety}

Chronic pain in COPD patients varies from $44 \%$ to $88 \%$, with an inverse relation to the health-related quality of life (HRQoL) $[24,29]$. The greater the presence of pain, the lower is the physical activity performed by the patient, negatively affecting the survival rate [24]. Chronic pain is associated with depression and greater anxiety, compared to people who have no chronic pain, and with a more altered postural attitude (hyperkyphosis). Pain is one of the causes of re-hospitalization and worsening of symptoms, with increased mortality (Figure 1) [30].

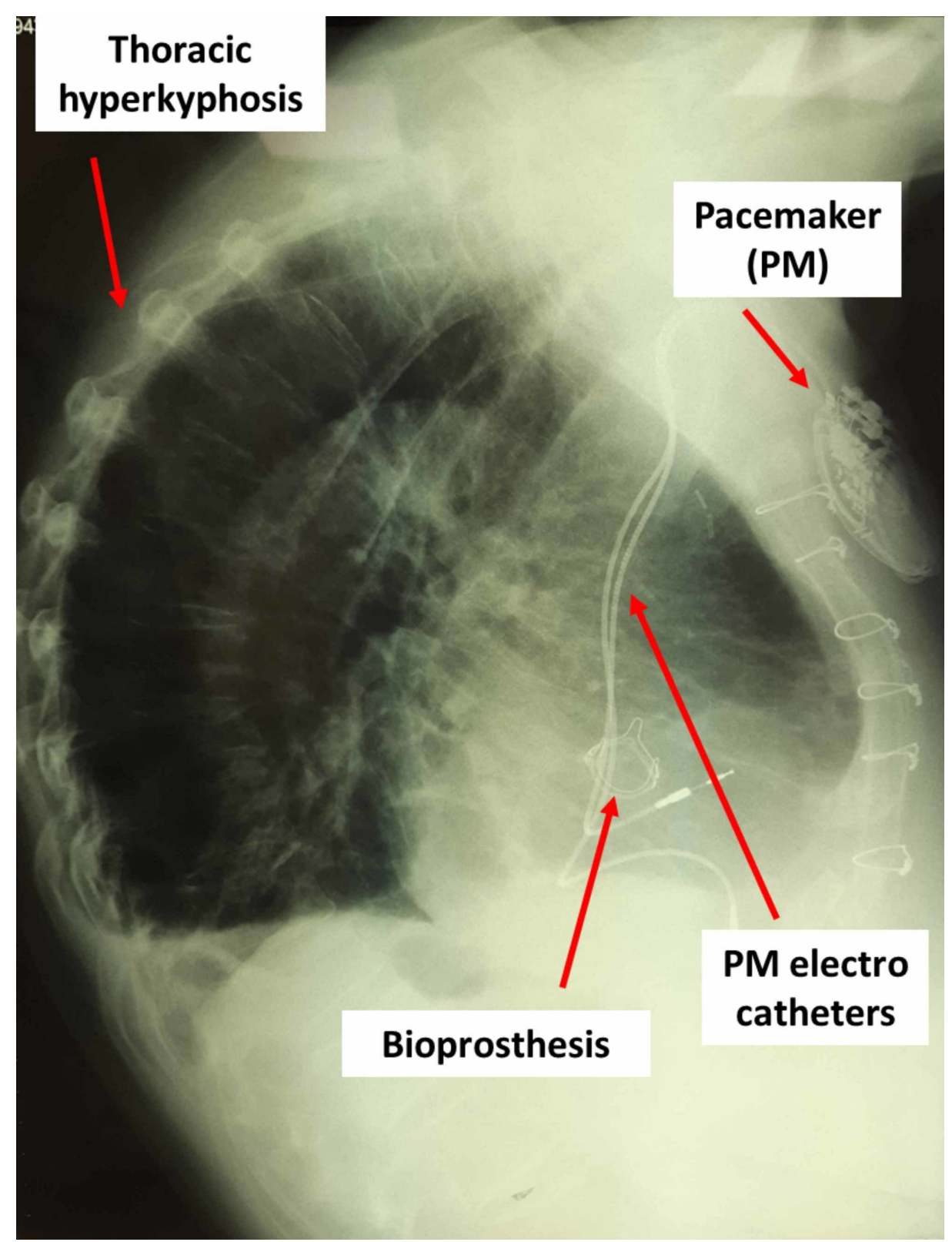

FIGURE 1: The patient with COPD shows an important dorsal hyperkyphosis, with a previous intervention in the median sternotomy for septal myectomy and aortic valve replacement with bioprosthesis, with pacemaker (PM) positioning

COPD, chronic obstructive pulmonary disease 
An altered emotional status, such as depression and anxiety, is one of the causes for the progression of lung disease [31]. They cause re-hospitalization, worsening of symptoms, and an increase in mortality [32,33]. The percentage of presence of depression and anxiety is around $40 \%$ and $36 \%$, respectively [32]. During the period of exacerbation of symptoms, depression rises in percentage (86\%), as does anxiety (55\%) [33]. Probably, the impact of the altered emotional status has a negative influence on the cognitive state.

\section{Effects of rehabilitation on chronic pain and on emotional status}

Pulmonary rehabilitation results in multiple benefits to the patient, although it can often be underappreciated compared to pharmacology [34]. The improvements may lead to changes in FEV1, functional capacity, QoL, and an improvement in the perception of pain [35]. Studies do not always directly evaluate the perception of pain, but it can be deduced from the improvement of different outcomes, as the QoL, the distance covered during the walking test; many patients suffer from chronic pathologies as osteoarthritis, presenting chronic pain [36,37]. There are several tests for the evaluation of pain, such as the Pain Sensitivity Questionnaire (PSQ), the Geriatric Pain Measure (GPM), and the Brief Pain Inventory (BPI) $[38,39]$. Chronic pain leads to depression and anxiety; furthermore, the fear of feeling pain leads to kinesiophobia, creating a dangerous closed circle [40]. Rehabilitation induces improvements in mood, as shown by the values of the Hospital Anxiety and Depression (HAD) test, with positive data up to 12 months post-training; the positive HAD score implements the patient's functional capacity [36]. Other data supporting the beneficial role of rehabilitation on depression and anxiety comes from the values measured by the Hamilton Rating Scale for Depression (HRSD) and the Hamilton Anxiety Scale (HAMA), the data they improve following intense activity and with patients with moderate and severe pathology [16]. The improvement of the emotional status increases the survival rates of the patient with COPD following training, even in people over 70 years of age, through the evaluation with the Beck Depression Inventory (BDI) [41].

\section{Cerebellum, emotions, and chronic pain}

Neuroimaging findings in COPD patients demonstrate structural alterations of the central nervous system [42]. The presence of chronic respiratory airway obstruction, particularly in smokers, is linked to the decrease in the cerebral gray matter; in patients with lower FEV1/FVC there is a volumetric decrease in white matter [42]. These adaptations are not necessarily linked to advancing age; moreover, these alterations reflect higher depression and anxiety values. Many studies show specific alterations, such as gray matter involved in the management of emotions (medial prefrontal cortex; anterior insula; amygdala; anterior cingulate cortex; hippocampus); other studies reveal generalized white matter lesions (myelin rarefaction, astrogliosis, axonal decrease and enlargement of perivascular spaces) [43,44]. A brain area negatively affected by the presence of COPD is the cerebellum [45]. The cerebellum sorts and manages peripheral information from the receptors related to the sensations of pain, interception, and exteroception (together with the vestibular area); this sensory information allows our brain to perceive how we are in the space in which we live, creating the motor and emotional patterns to interact. The cerebellum modulates behavioral and pain homeostasis. There is a close relationship between altered movement and behavioral and pain perception imbalances. The pain information arrives in the lobules IV-X and crus I-II (posterior area of the cerebellum), as the information arrives (from the return) also from the cortex (frontal, temporal, and parietal); the aforementioned cerebellar areas are activated even before experiencing pain [46]. The nociceptive information that reaches the cerebellum is sorted and "translated" towards the centers of the cortex and the centers of the limbic area, in a bi-univocal manner. Chronic pain reduces the size of the areas to which nociceptive information arrives [46]. The nociceptive afferent pathways travel via the spinoolivocerebellar pathway, involving the climbing fibers, the Purkinje cells; the latter also receive visceral information in the posterior cerebellar area (vermis). Pain circuits involve emotional and cognitive, motor and perceptual status [47]. Motor movement is the result of the response that the cerebellum, after it has filtered from the limbic, vestibular area, from the area of the cortex and from the entire whole/exteroceptive system, a lot of information. If, as in the case of patients with COPD, the cerebellum undergoes structural and functional alterations, there will be motor disorders connected to an incorrect perception of the nociceptive stimulus and depression [47]. As regards the cerebellum and emotions, a branch of neurological medicine names the cerebellar non-motor function that influences depression and anxiety as an "emotional cerebellum" [48]. The cerebellum processes negative and positive emotions. If there is a cerebellar lesion, negative emotions are not processed correctly (in synergy with the right hemisphere) and this will lead to aberrant emotional behaviors, such as depression [48]. The neurological circuit of depression and anxiety connects the cerebellum to different brain areas: the amygdala, hippocampus, turnip nuclei, periaqueductal gray area, pre-frontal cortical area, ventral tegmental area, and dopaminergic areas. The cerebellar areas involved in the reception of emotional and behavioral status, such as anxiety and depression, are lobules VI and VII in the neocerebellar vermis (also known as the limbic area of the cerebellum). When the patient suffers from depression and anxiety, most likely there is an alteration of the cerebellar function (hyperactivity or hypoactivity) and a reduced collaboration with the areas of the brain mentioned above. These latter brain areas have a one-to-one connection with the cerebellum. Patients entities with COPD show cerebral and structural metabolic changes, including the cerebellum; these alterations limit the information network between the different areas of the central nervous system [45].

\section{The proprioceptivity in COPD patients}


We know that there is a close relationship between movement alteration (coordination) and emotional status, as well as the processing of physical pain. We know that the COPD patient undergoes an alteration of the morphology and function of the cerebellum, as well as the important function of the cerebellar area in the management of non-motor functions is known. The cerebellum plays a primary role in managing the proprioceptive information, fundamental, among other functions, for the proper execution of the movement while maintaining balance. COPD patients show a reduction in proprioception, which condition results in less body control during daily movement with, for example, a weaker postural maintenance capacity of the ankle; the proprioceptive afferents coming from the tissues (muscles and connective tissue) are unable to be adequately processed by the cerebellum or have lost part of their role, or both conditions [30]. Inserting exercises to improve proprioception in COPD patients should be a usual procedure, through balance training, in the light of the reflections shown in the current text and for the reduced proprioceptive capacity. Studies that included balance training in the rehabilitation process (BT) showed improvements in balance and posture, indicators of improved proprioception $[49,50]$ We do not yet have sufficient data to recommend the appropriate amount and method of administration of BT in this category of patients. Probably, the best age to administer BT is after 50 years of age, as motor imbalances are more evident from this age onwards. BT not only improves proprioceptive indices but could stimulate cerebellar areas to improve afference management body, which will be related to pain, depression, and anxiety. The pursuit of physical activity in the rehabilitation process improves motor coordination (and consequently proprioception), with improved emotional and pain threshold status $[36,39,41]$. Adding a specific stimulation for the cerebellar area (BT) could be a useful strategy for the implementation of the patient's well-being with COPD, to be combined with the usual rehabilitation process. Currently, there are no specific data with large numbers on the relationship of the insertion of BT in conjunction with the pulmonary rehabilitation process. Further studies are awaited.

\section{Conclusions}

COPD patients suffer from various comorbidities, including a lower pain threshold and chronic pain, depression and anxiety. The quality of movement, understood as balance, posture and proprioceptive quality, has decreased. Studies show structural and functional alterations of the central nervous system in patients, including the cerebellum and cerebellar connections (afferents and efferences). There is a close relationship between cerebellar changes, motor changes, and the presence of non-physiological emotional status. The article has reviewed the literature concerning the possible influences of cerebellar aberrations and some comorbidities of patients with chronic lung diseases, hypothesizing to put the use of balance training in the usual rehabilitation process. The latter approach should stimulate the cerebellar areas more specifically and improve the symptomatic picture, with a wider clinical gain. Further and wider researches are needed.

\section{Additional Information \\ Disclosures}

Conflicts of interest: In compliance with the ICMJE uniform disclosure form, all authors declare the following: Payment/services info: All authors have declared that no financial support was received from any organization for the submitted work. Financial relationships: All authors have declared that they have no financial relationships at present or within the previous three years with any organizations that might have an interest in the submitted work. Other relationships: All authors have declared that there are no other relationships or activities that could appear to have influenced the submitted work.

\section{References}

1. Gao H, Gao Y, Sun P, Shen J, Yao HJ, Fu SD, Meng C: Effect of physical therapy for chronic obstructive pulmonary disease: a protocol for an updated systematic review of randomized controlled trial. Medicine (Baltimore). 2019, 98:e17241. 10.1097/MD.0000000000017241

2. Araujo ZTS, Mendonça KMPP, Souza BMM, Santos TZM, Chaves GSS, Andriolo BNG, Nogueira PAMS: Pulmonary rehabilitation for people with chronic obstructive pulmonary disease: A protocol for an overview of Cochrane reviews. Medicine. 2019, 98:e17129. 10.1097/MD.0000000000017129

3. Zhang L, Huang J, Dong R, Feng Y, Zhou M: Therapeutic potential of BLT1 antagonist for COPD: involvement of inducing autophagy and ameliorating inflammation. Drug Des Devel Ther. 2019, 13:31053116. 10.2147/DDDT.S215433

4. Gershon AS, Thiruchelvam D, Aaron S, et al.: Socioeconomic status (SES) and 30-day hospital readmissions for chronic obstructive pulmonary (COPD) disease: a population-based cohort study. PLoS One. 2019, 14:e0216741. 10.1371/journal.pone.0216741

5. Mostafaei S, Kazemnejad A, Azimzadeh Jamalkandi S, et al.: Identification of novel genes in human airway epithelial cells associated with chronic obstructive pulmonary disease (COPD) using machine-based learning algorithms. Sci Rep. 2018, 8:15775. 10.1038/s41598-018-33986-8

6. Buhr RG, Jackson NJ, Kominski GF, Dubinett SM, Ong MK, Mangione CM: Comorbidity and thirty-day hospital readmission odds in chronic obstructive pulmonary disease: a comparison of the Charlson and Elixhauser comorbidity indices. BMC Health Serv Res. 2019, 19:701. 10.1186/s12913-019-4549-4

7. Iyer AS, Bhatt SP, Garner JJ, et al.: Depression is associated with readmission for acute exacerbation of chronic obstructive pulmonary disease. Ann Am Thorac Soc. 2016, 13:197-203. 10.1513/AnnalsATS.201507$4390 \mathrm{C}$

8. Bordoni B, Marelli F, Morabito B, Sacconi B: Manual evaluation of the diaphragm muscle . Int J Chron 
Obstruct Pulmon Dis. 2016, 11:1949-1956. 10.2147/COPD.S111634

9. Bordoni B, Marelli F, Morabito B, Castagna R: Chest pain in patients with COPD: the fascia's subtle silence . Int J Chron Obstruct Pulmon Dis. 2018, 13:1157-1165. 10.2147/COPD.S156729

10. Lal C, Kumbhare S, Strange C: Prevalence of self-reported sleep problems amongst adults with obstructive airway disease in the NHANES cohort in the United States [Epub ahead of print]. Sleep Breath. 2019, 10.1007/s11325-019-01941-0

11. Samarghandi A, Ioachimescu OC, Qayyum R: Association between peak inspiratory flow rate and hand grip muscle strength in hospitalized patients with acute exacerbation of chronic obstructive pulmonary disease. PLoS One. 2020, 15:e227737. 10.1371/journal.pone.0227737

12. Sahin H, Naz I, Susam S, Erbaycu AE, Olcay S: The effect of the presence and severity of bronchiectasis on the respiratory functions, exercise capacity, dyspnea perception, and quality of life in patients with chronic obstructive pulmonary disease. Ann Thorac Med. 2020, 15:26-32. 10.4103/atm.ATM_198_19

13. Gupta A, Ravaliya V, Mishra D, Dani V, Sodawala C, Shah H, Patel D: Assessment of knowledge, attitude, and behavior about the disease process and physiotherapy management in patients with chronic obstructive pulmonary disease: a qualitative study. J Educ Health Promot. 2019, 8:15. 10.4103/jehp.jehp_209_18

14. McCarthy B, Casey D, Devane D, Murphy K, Murphy E, Lacasse Y: Pulmonary rehabilitation for chronic obstructive pulmonary disease. Cochrane Database Syst Rev. 2015, CD003793. 10.1002/14651858.CD003793.pub3

15. Spruit MA, Singh SJ, Garvey C, et al.: An official american thoracic society/european respiratory society statement: key concepts and advances in pulmonary rehabilitation. Am J Respir Crit Care Med. 2013, 188:e13-e64. 10.1164/rccm.201309-1634ST

16. He GX, Li N, Ren L, et al.: Benefits of different intensities of pulmonary rehabilitation for patients with moderate-to-severe COPD according to the GOLD stage: a prospective, multicenter, single-blinded, randomized, controlled trial. Int J Chron Obstruct Pulmon Dis. 2019, 14:2291-2304. 10.2147/COPD.S214836

17. Spruit MA: Pulmonary rehabilitation. Eur Respir Rev. 2014, 23:55-63. 10.1183/09059180.00008013

18. Aquino G, Iuliano E, di Cagno A, et al.: Effects of combined training vs aerobic training on cognitive functions in COPD: a randomized controlled trial. Int J Chron Obstruct Pulmon Dis. 2016, 11:711-718. 10.2147/COPD.S96663

19. Rugbjerg M, Iepsen UW, Jørgensen KJ, Lange P: Effectiveness of pulmonary rehabilitation in COPD with mild symptoms: a systematic review with meta-analyses. Int J Chron Obstruct Pulmon Dis. 2015, 10:791801. 10.2147/COPD.S78607

20. Porto EF, Castro AA, Schmidt VG, Rabelo HM, Kümpel C, Nascimento OA, Jardim JR: Postural control in chronic obstructive pulmonary disease: a systematic review. Int J Chron Obstruct Pulmon Dis. 2015, 10:1233-1239. 10.2147/COPD.S63955

21. Beauchamp MK: Balance assessment in people with COPD: an evidence-based guide . Chron Respir Dis. 2019, 16:1479973118820311. 10.1177/1479973118820311

22. Roig M, Eng JJ, Road JD, Reid WD: Falls in patients with chronic obstructive pulmonary disease: a call for further research. Respir Med. 2009, 103:1257-1269. 10.1016/j.rmed.2009.03.022

23. Morlino P, Balbi B, Guglielmetti S, et al.: Gait abnormalities of COPD are not directly related to respiratory function. Gait Posture. 2017, 58:352-357. 10.1016/j.gaitpost.2017.08.020

24. Lee AL, Goldstein RS, Brooks D: Chronic pain in people with chronic obstructive pulmonary disease: prevalence, clinical and psychological implications. Chronic Obstr Pulm Dis. 2017, 4:194-203. 10.15326/jcopdf.4.3.2016.0172

25. Mills SEE, Nicolson KP, Smith BH: Chronic pain: a review of its epidemiology and associated factors in population-based studies. Br J Anaesth. 2019, 123:273-283. 10.1016/j.bja.2019.03.023

26. Bordoni B, Marelli F, Morabito B, Sacconi B, Caiazzo P, Castagna R: Low back pain and gastroesophageal reflux in patients with COPD: the disease in the breath. Int J Chron Obstruct Pulmon Dis. 2018, 13:325-334. 10.2147/COPD.S150401

27. Weinreich UM, Thomsen LP, Bielaska B, Jensen VH, Vuust M, Rees SE: The effect of comorbidities on COPD assessment: a pilot study. Int J Chron Obstruct Pulmon Dis. 2015, 10:429-438. 10.2147/COPD.S76124

28. Bordoni B, Marelli F, Morabito B, Sacconi B: Depression, anxiety and chronic pain in patients with chronic obstructive pulmonary disease: the influence of breath. Monaldi Arch Chest Dis. 2017, 87:811. 10.4081/monaldi.2017.811

29. van Dam van Isselt EF, Groenewegen-Sipkema KH, van Eijk M, Chavannes NH, Achterberg WP: Pain in patients with chronic obstructive pulmonary disease indicated for post-acute pulmonary rehabilitation. Chron Respir Dis. 2019, 16:1479972318809456. 10.1177/1479972318809456

30. Vardar-Yagli N, Saglam M, Calik-Kutukcu E, Inal-Ince D, Arikan H, Coplu L: Increased pain sensitivity, postural abnormalities, and functional balance impairment in obstructive lung disease compared to healthy subjects. Heart Lung. 2019, 48:351-355. 10.1016/j.hrtlng.2018.12.009

31. Rzadkiewicz M, Nasiłowski J: Psychosocial interventions for patients with severe COPD-an up-to-date literature review. Medicina (Kaunas). 2019, 55:597. 10.3390/medicina55090597

32. Smith SM, Sonego S, Ketcheson L, Larson JL: A review of the effectiveness of psychological interventions used for anxiety and depression in chronic obstructive pulmonary disease. BMJ Open Respir Res. 2014, 1:e000042. 10.1136/bmjresp-2014-000042

33. Li Z, Liu S, Wang L, Smith L: Mind-body exercise for anxiety and depression in COPD patients: a systematic review and meta-analysis. Int J Environ Res Public Health. 2019, 17:22. 10.3390/ijerph17010022

34. Bhatt SP: It's time to rehabilitate pulmonary rehabilitation. Ann Am Thorac Soc. 2019, 16:55-57. 10.1513/AnnalsATS.201809-641ED

35. Schroff P, Hitchcock J, Schumann C, Wells JM, Dransfield MT, Bhatt SP: Pulmonary rehabilitation improves outcomes in chronic obstructive pulmonary disease independent of disease burden. Ann Am Thorac Soc. 2017, 14:26-32. 10.1513/AnnalsATS.201607-5510C

36. Grosbois JM, Gicquello A, Langlois C, Le Rouzic O, Bart F, Wallaert B, Chenivesse C: Long-term evaluation of home-based pulmonary rehabilitation in patients with COPD. Int J Chron Obstruct Pulmon Dis. 2015, 10:2037-2044. 10.2147/COPD.S90534 
37. Ryrsø CK, Godtfredsen NS, Kofod LM, et al.: Lower mortality after early supervised pulmonary rehabilitation following COPD-exacerbations: a systematic review and meta-analysis. BMC Pulm Med. 2018, 18:154. 10.1186/s12890-018-0718-1

38. Lewthwaite H, Williams G, Baldock KL, Williams MT: Systematic review of pain in clinical practice guidelines for management of COPD: a case for including chronic pain?. Healthcare (Basel). 2019, 7:15. 10.3390/healthcare7010015

39. Johnson AM, Smith SM: A review of general pain measurement tools and instruments for consideration of use in COPD clinical practice. Int J Chron Obstruct Pulmon Dis. 2017, 12:923-929. 10.2147/COPD.S119889

40. Vardar-Yagli N, Calik-Kutukcu E, Saglam M, Inal-Ince D, Arikan H, Coplu L: The relationship between fear of movement, pain and fatigue severity, dyspnea level and comorbidities in patients with chronic obstructive pulmonary disease. Disabil Rehabil. 2019, 41:2159-2163. 10.1080/09638288.2018.1459886

41. Alsaraireh FA, Aloush SA: Does pulmonary rehabilitation alleviate depression in older patients with chronic obstructive pulmonary disease. Saudi Med J. 2017, 38:491-496. 10.15537/smj.2017.5.17965

42. Spilling CA, Bajaj MK, Burrage DR, et al.: Contributions of cardiovascular risk and smoking to chronic obstructive pulmonary disease (COPD)-related changes in brain structure and function. Int J Chron Obstruct Pulmon Dis. 2019, 14:1855-1866. 10.2147/COPD.S213607

43. Esser RW, Stoeckel MC, Kirsten A, et al.: Brain activation during perception and anticipation of dyspnea in chronic obstructive pulmonary disease. Front Physiol. 2017, 8:617. 10.3389/fphys.2017.00617

44. Kim KW, MacFall JR, Payne ME: Classification of white matter lesions on magnetic resonance imaging in elderly persons. Biol Psychiatry. 2008, 64:273-280. 10.1016/j.biopsych.2008.03.024

45. Li H, Xin H, Yu J, Yu H, Zhang J, Wang W, Peng D: Abnormal intrinsic functional hubs and connectivity in stable patients with COPD: a resting-state MRI study. Brain Imaging Behav. 2019, 14:573-585. 10.1007/s11682-019-00130-7

46. Michelle Welman FHS, Smit AE, Jongen JLM, Tibboel D, van der Geest JN, Holstege JC: Pain experience is somatotopically organized and overlaps with pain anticipation in the human cerebellum. Cerebellum. 2018, 17:447-460. 10.1007/s12311-018-0930-9

47. Moulton EA, Schmahmann JD, Becerra L, Borsook D: The cerebellum and pain: passive integrator or active participator?. Brain Res Rev. 2010, 65:14-27. 10.1016/j.brainresrev.2010.05.005

48. Adamaszek M, D'Agata F, Ferrucci R, et al.: Consensus paper: cerebellum and emotion. Cerebellum. 2017, 16:552-576. 10.1007/s12311-016-0815-8

49. Mkacher W, Mekki M, Tabka Z, Trabelsi Y: Effect of 6 months of balance training during pulmonary rehabilitation in patients with COPD. J Cardiopulm Rehabil Prev. 2015, 35:207-213. 10.1097/HCR.0000000000000109

50. Hill K: Balance-specific training embedded within a pulmonary rehabilitation program may reduce falls risk in people with COPD. J Physiother. 2014, 60:111. 10.1016/j.jphys.2014.05.013 